\title{
Investigation of the User Experience and Effects of Compression on the Body
}

\section{Esther Foo}

Human Factors and Ergonomics

University of Minnesota-Twin Cities

St Paul, MN 55108, USA

efoo@umn.edu

\begin{abstract}
This paper outlines an ongoing PhD dissertation research, seeking to explore the user experience of a non-traditional form of haptic modality - compression, through the use of computer-mediated on-body compression systems with embedded active materials that are capable of being simultaneously functional, remotely-controllable, and inconspicuous. The motivation for this work is to address the need to expand our understanding of different haptic sensations, in this case, the objective and subjective effects of compression on a user, as well as how might the use of active, dynamically-controllable garments affect traditional compression garment use paradigms.
\end{abstract}

\section{Author Keywords}

Wearable Technology; Affective Haptics; Haptic

Communication; Wearable Haptics; Human Factors

personal or classroom use is granted without fee provided that copies are personal or classroom use is granted without fee provided that copies are bear this notice and the full citation on the first page. Copyrights for thirdparty components of this work must be honored. For all other uses, contact the Owner/Author.

UbiComp/ISWC'18 Adjunct, October 8-12, 2018, Singapore, Singapore (C) 2018 Copyright is held by the owner/author(s).

ACM ISBN 978-1-4503-5966-5/18/10.

https://doi.org/10.1145/3267305.3278483

\section{ACM Classification Keywords}

H.1.2. User/Machine Systems: Human factors; H.5.2.

Information Interfaces and Presentation: User

Interfaces- Haptic $I / O$ 


\section{Research Topic}

The sense of touch allows us to receive information about the internal/external environment. One of the sensations that can be detected is compression. Compression is present in many facets of our lives; from the ubiquitous (e.g., wearing clothing), to the emotional (e.g., a hug), and even healthcare applications (e.g., compression stockings to improve blood circulation [1]). Interventions such as infant swaddling, massage therapies, and weighted vests, all benefit from the use of compression to positively affect the wellbeing and emotion of users by promoting relaxation and decreasing anxiety [2], [3].

Compression is also currently being explored as a form of haptic modality, since it is less attention-demanding (compared to vibrotactile display that can be disruptive when prolonged) [4], able to invoke a wide range of attention capture (subtle to forceful), is similar to common human behaviors (e.g., a hug), carries huge emotional significance [5], and is known to improve the physical and emotional well-being of users (e.g., deep pressure therapy for children with sensory processing difficulties) [2].

Current research using compression intersects many fields such as $\mathrm{HCI}$, affective haptics, and occupational therapy (OT) [5]-[7]. However, several research gaps still remain unexplored. The first being to truly maximize the use of compression in a variety of applications, the effects of compression on the human body, must be better understood. Broadly, the experience of compression can be modulated with varying inputs including compression location, intensity, rate, and duration. However, there is a lack of rigorous study with respective to stimulation for a desired experience. Further, there is a lack of focus on using a garment form factor to distribute compressive forces onto larger areas of the body (i.e., the torso). The garment platform is ideal for creating compression due to the close proximity to the body, large direct-acting surface area, as well as the social ubiquity of clothing, provides a physically intimate yet low-profile solution. Current garment-based compression systems are either (1) passive (non-adjustable)- compression through added weights or stretch (e.g. elastic clothing, weighted vests), or (2) dynamic- constricting the user. However, both designs suffer from functional or usability issues, such as limitations in donning/ doffing, mobility, portability, and bulk [8]. Further, one of the main deterrents that prevents rigorous study of these problems is the lack of ability of current garment solutions to precisely/dynamically control the required compression generated. A potential solution is to develop computer-mediated compression garments that are dynamic, low-profile, and remotely-controllable. The key idea is to create an innovative garment platform that abandons traditional wearable actuation schemes (e.g. servos, pneumatics) and instead shifts toward the use of embedded active materials to produce actively controllable compression. Here, shape memory alloy (SMA) coiled actuators are used given its prior success in producing controllable compression $(<225 \mathrm{mmHg})$ that scales with applied current [8]. Therefore, this paper outlines an ongoing PhD project, seeking to investigate the use of computer-mediated on-body compression systems to expand our understanding of the objective and subjective effects of compression on a user.

\section{Research Methodology}

This work is divided into three phases. Starting with the broad approach of understanding how users experience 


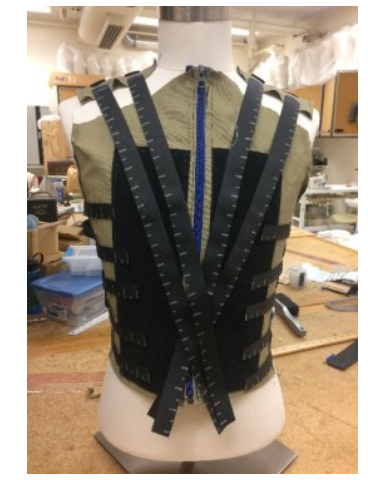

Figure 1: Passive Garment

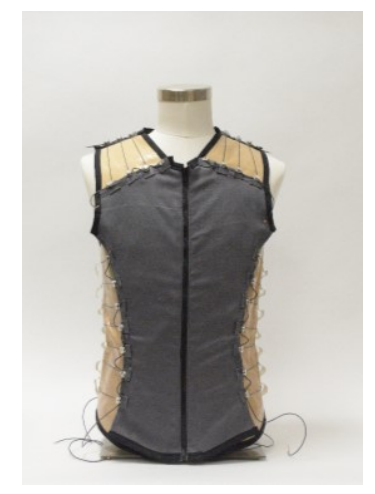

Figure 2: SMA-based Active Compression Garment (front view) compression on various areas on the upper body, followed by the investigation of the variables mediating the experience of compression. Once the user experiences are better understood, the system will be deployed to judge its effectiveness at modulating user affect. All studies will use a healthy adult (ages 18-34) target population.

Phase 1 of this research focuses on evaluating the feasibility of producing a computer-mediated active compression garment system. Two pilot studies will be conducted. First, an adjustable passive compression garment will be created using readily available materials (e.g. hook and loop fasteners and passive straps to apply adjustable compression). Second, a dynamic compression garment incorporating SMAbased active materials as a compression actuation mechanism will be developed. Both the passive and active garments will be used in small-scale user studies to qualitatively evaluate subjective experiences of users when exposed to variable upper-body compression.

Phase 2 investigates the effects of compression given varying inputs. The experience of compression will be modulated through variable location, rate, duration, and intensity profiles, using an SMA-based garment architecture, equipped with on-board power systems and wireless control. The effects of these inputs will be measured objectively (e.g., heart rate (HR), electrodermal responses (EDA), respiration rate (RR)) and subjectively (through subjective user interview responses and comfort/discomfort thresholds). The knowledge gained from this study will be used to finetune the operating paradigms in computer-mediated compression. The study will be further divided into stationary (i.e., seated with minimal movement) and mobile (i.e., walking), to ensure that compression is still perceivable when subjects are in motion (important in actual use scenario).

Phase 3 will involve a larger scale context-specific user study of computer-mediated compression. Since evidence in the literature has pointed to the ability of compression to induce calming effects, an experiment would be one that employs an arousal-inducing scenario such as the Stroop color word test, a wellestablished sympathetic response-inducing method [9]. The idea here is to investigate if compression can be used to 'calm' a user, an application that has many implications in the field of OT. As before, both objective/subjective measures will be collected. It is hypothesized that compression will provide reduced physiological arousal (decreased HR, RR, EDA) and reduced arousal in subjective self-report responses compared to the control.

\section{Research Completed So Far}

Currently, Phase 1 research has been completed, with a preliminary user study $(n=5)$ for the passive garment (Figure 1$)$ and $(n=8)$ for the SMA-based active garment (Figures 2-4).

Both studies from Phase 1 demonstrate that it was feasible to use integrated SMA to selectively apply compression on the body. Participants generally reacted positively to compression. Some common themes in the qualitative interview involved feelings of calming/comforting/warm, and is suggested to be potentially useful in health contexts (exercise or stressrelief). These preliminary results show that there is potential in the use of distributed compression on large areas of the body as an interaction modality, since it is 


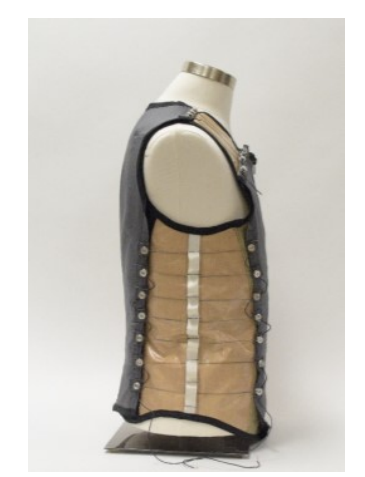

Figure 3: SMA-based active compression garment (side view)

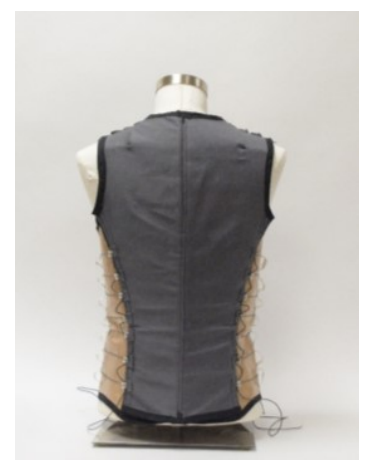

Figure 4: SMA-based active compression garment (back view) detectable, welcomed if done correctly, and can be induced within a low-profile garment form factor. The study provided insight into the subjective effects of onbody compression and the active garment has significant potential to be used as an evaluation tool to further study compression as a haptic modality.

\section{Conclusion}

This work seeks to understand the comfort and emotional effects of upper-body compression; the major contributions include:

(1) Advancement of wearable active compression systems that will serve as a platform for future investigations.

(2) Understand the objective and subjective effects of computer-mediated on-body compression and its ability in affect mediation.

(3) Understand how might the use of active compression garments affect traditional compression

\section{garment use paradigms.}

\section{Acknowledgements}

This work is supported by NSF grant \# 1656995.

\section{Biographical Sketch}

Esther Foo is a third year doctoral candidate in Human Factors and Ergonomics at the University of Minnesota. She started the program in September of 2016 and is expected to graduate in May 2020. She obtained her B.Sc. degree in Biomedical Engineering from Purdue University, M.Eng. degree in Biomedical Engineering from Cornell University, and M.Sc. degree in Human Factors and Ergonomics from the University of Minnesota. Esther's research interest lies in the human factors aspects of medical/healthcare applications and wearable systems. Her specific PhD dissertation research focuses on the objective and subjective effects of computer-mediated compression on a user, in the hopes of contributing to cross-cutting research areas including wearable technology, affective haptics, haptic interfaces, and human-computer interaction (HCI). Esther is advised by Dr. Brad Holschuh, an Assistant Professor of Wearable Technology and Apparel Design at the University of Minnesota and holds additional appointments in Aerospace Engineering/Mechanics, Human Factors/Ergonomics, and Design.

\section{Statement of Objectives}

Other than having the privilege to present my work in this premier conference and broaden my knowledge base, I also see this as a wonderful opportunity to grow my professional network. Since the field of wearable computing is still relatively new, it is rare to come across conferences that are specifically narrowed down to align with my research interests. Having the opportunity to connect with other researchers in the field will be an invaluable experience. I am also especially excited about learning more about how to better communicate my work within and beyond the academic community; it is an excellent chance to gain inspiration from other fellow researchers. But perhaps more importantly, I relish the opportunity to hear about other people's experience working in this field, especially from the diverse group of anticipated audience. Since my research interests lie at the intersection of multiple research fronts, I have always believed that diversity, being a catalyst to innovation in the growing field of design, is essential to driving any technology forward. I truly appreciate cross-cultural understanding and am constantly looking for opportunities to develop myself in those areas. 


\section{References}

1. L. Macintyre and M. Baird, "Pressure garments for use in the treatment of hypertrophic scars," Burns, vol. 31, no. 1, pp. 11-14, 2005.

2. T. Grandin, "Calming effects of deep touch pressure in patients with autistic disorder, college students, and animals.," J. Child Adolesc. Psychopharmacol., vol. 2, no. 1, pp. 63-72, 1992.

3. T. Field, Touch. Cambridge, MA: MIT Press, 2003.

4. H. Pohl, et .al., "Squeezeback: Pneumatic Compression for Notifications," Proc. SIGCHI Conf. Hum. Factors Comput. Syst. - CHI '17, 2017.

5. F. "Floyd" Mueller, F. Vetere, M. R. Gibbs, J. Kjeldskov, S. Pedell, and S. Howard, "Hug over a distance," Hum. factors Comput. Syst. - CHI '05, p. 1673

6. H. Pohl, F. Hoheisel, and M. Rohs, "Inhibiting Freedom of Movement with Compression Feedback," Proc. 2017 CHI EA '17, pp. 1962-1969, 2017.

7. S. Reynolds, S. J. Lane, and B. Mullen, "Effects of Deep Pressure Stimulation on Physiological Arousal," Am. J. Occup. Ther., vol. 69, 2015.

8. B. Holschuh and D. Newman, "Two-spring model for active compression textiles with integrated $\mathrm{NiTi}$ coil actuators," Smart Mater. Struct., vol. 24, no. 3,

9. J. H. M. Tulen, et. al., "Characterization of stress reactions to the Stroop Color Word Test," Pharmacol. Biochem. Behav., vol. 32, no. 1, pp. 9$15,1989$. 